\title{
Parallel Mappings as a Key for Understanding the Bioinorganic Materials
}

\author{
A. Kuczumow ${ }^{a, *}$, J. NowaK ${ }^{a}$ And R. Chąas ${ }^{b}$ \\ ${ }^{a}$ Department of Chemistry, John Paul II Catholic University of Lublin \\ Kraśnicka 102, 20-718 Lublin, Poland \\ ${ }^{b}$ Department of Conservative Dentistry, Medical University of Lublin \\ Karmelicka 7, 20-081 Lublin, Poland
}

\begin{abstract}
Important bioinorganic objects, both living and fossilized are as a rule characterized by a complex microscopic structure. For biological samples, the cell-like and laminar as well as growth ring structures are among most significant. Moreover, these objects belong to a now widely studied category of biominerals with composite, inorganic-organic structure. Such materials are composed of a limited number of inorganic compounds and several natural organic polymers. This apparently simple composition leads to an abnormal variety of constructions significant from the medical (repairs and implants), natural (ecological effectiveness) and material science (biomimetic synthesis) point of view. The analysis of an image obtained in an optical microscope, optionally in a scanning electron microscope is a topographical reference for further investigations. For the characterization of the distribution of chemical elements and compounds in a material, techniques such as X-ray, electron- or proton microprobes are applied. Essentially, elemental mappings are collected in this stage. The need for the application of an X-ray diffraction microprobe is obvious and our experience indicates on the necessity of using the synchrotron-based devices due to their better spatial resolution and good X-ray intensity. To examine the presence of the organic compounds, the Raman microprobe measurements are good options. They deliver information about the spatial distribution of functional groups and oscillating fragments of molecules. For the comprehensive investigation of bioinorganic material structural and chemical features, we propose the following sequence of methods: optical imaging, elemental mapping, crystallographic mapping, organic mapping and micromechanical mapping. The examples of such an approach are given for: petrified wood, human teeth, and an ammonite shell.
\end{abstract}

PACS numbers: 78.70.En, 87.64.Bx, 87.64.Dz, 87.59.-e, 82.80.-d

\section{Introduction}

Inorganic biomaterials attract more and more attention among scientists and in the real life applications $[1,2]$. They are common skeletal materials in the nature. It is surprising but the number of inorganic compounds belonging to this category is very limited $[3,4]$. Two crystallographic forms of calcium carbonate, varieties of silica and apatites are nearly exclusive materials, with calcium constituting nearly $50 \%$ of cations and phosphates making up about $25 \%$ of anions participating in biomineralization processes. The calcium carbonate and silica are present in skeletons of lower animals and apatites essentially form skeletons and teeth of the vertebrates. The total number of inorganic species involved in biomaterials does not exceed 80. Although popularly known as inorganic biomaterials, they significantly differ from their simpler, mineralogical analogues. First of all, they always include organic compounds. Most often we find natural organic polymers such as cellulose, lignin,

\footnotetext{
* corresponding author; e-mail: kuczo@kul.lublin.pl
}

chitin, collagen. Some of those polymers have many different forms (e.g. collagen, there are at least 20 its varieties) and the other are not well defined (lignin is here a good example). In many structures organic and inorganic components are intertwined or the major inorganic component is organized on the minor organic template. This kind of organization gives very surprising results. The new materials have features which are very different from these of the component materials. Objects built of hard inorganic materials become much more elastic after the inclusion of an organic component [5] and the shapes of objects formed of such materials can be very unusual. The sophisticated spiral shapes of shells of different mussels are good examples of such forms. Moreover, such forms are also discovered in different fossils, which preserved original shapes of animal skeletons for many millions years. The recent studies on biomaterials are aimed at different directions. The recognition of their structure gives us information about the organization of structural elements of our bodies or bodies of animals and plants. It leads to the efficient treatment of diseases or to the construction of prostheses. Other researchers 
fascinated by the achievements of the nature aim their efforts at biomimetic syntheses of materials of unique features. Since the construction of natural biomaterials is highly organized, most often structure ordering is laminar, ring or cell-like, then their periodic patterns convey information on structure growth and may deliver information on the conditions in which the growth took place. If the patterned structure is built for some time, the external parameters such as temperature or humidity are likely to influence its ordering and chemical composition. Thus, the deciphering of the information included in the periodic patterns helps to reconstruct the climatic and environmental conditions of the distant past. The studies on the extraordinary stability of fossils formed of such materials should also be carried out. We sometimes look for "everlasting" materials, which can prevent dangerous wastes from releasing to the environment for a long time and the examination of fossils may be extremely educative.

To be able to observe and study such objects, one should have relevant tools. A micrometer size is one of the essential dimensions observed in the structure of biomaterial samples. The size of biological cells is of the order of micrometers or tens of micrometers. Some subcellar structures are of micrometer size. The dimensions of laminar structures are often below one hundred micrometers. Thus, only microprobes with the spatial resolution of the order of micrometer are the proper devices for examination of biomaterial structure features. Analyzing bioinorganic objects one should pay attention to:

1. optical appearance of a structure;

2. elemental composition;

3. phase (most often crystallographic) composition;

4. organic components, very often being a template;

5. mechanical characteristics;

6. surface features.

The optical observation followed by the transformation of obtained images to digital files, which can be easily stored, is an essential stage. It reveals the morphology of a sample. As we discussed above, bioinorganic samples are patterned and thus all subsequent measurement results can be related to the fragments of the patterns. It is of great instructive value to relate chemical, structural, organic and mechanical features of a sample to the topographic details in an optical image. It leads to the identification and understanding how the bioinorganic object functions.

\section{Instrumentation}

Spatial elemental analyses can be performed using such techniques as micro X-ray fluorescence spectrometry $(\mu$-XRF) in tabletop or synchrotron-based versions; electron probe micro analysis (EPMA); or micro particle induced X-ray emission ( $\mu$-PIXE). Although they deliver similar information, they are not identical. Anyone selecting a method who must compromise between the need for good spatial resolution, ability to detect very light elements, and immediate coupling with secondary/backscatter electron image will likely choose EPMA, good minimum detection limits and ability to detect trace elements ensure PIXE, good depth penetration - XRF and great beam intensity — synchrotron-based XRF.

In our study of biominerals, described in this paper, we used a scanning electron microscope LEO $1430 \mathrm{VP}$ at the Catholic University of Lublin with a $\mathrm{Si}(\mathrm{Li})$ detector (Röntec) with an energy resolution of $180 \mathrm{eV}$, as measured for $\mathrm{Mn} K_{\alpha}$ line. This instrument is described in more detail in the paper by Kuczumow et al. [6]. The $\mathrm{XRF}$ device used is a synchrotron version, it operates at the $L$ line in HASYLAB and was described by Falkenberg et al. [7]. The application of PIXE is not disputed in this paper. A good representative version of the instrument is at the Niewodniczański Institute of Nuclear Physics in Cracow, it was described by Lebed et al. [8].

The microdiffraction device used here was the MAXIM facility in HASYLAB $[9,10]$. Although we preferred the much simpler device built by Chevallier et al. [11, 12] at the now closed LURE, we applied Hamburg's facility accepting its not so encouraging spatial resolution $(>12 \mu \mathrm{m})$ as this drawback was partially compensated by the possibility of performing relatively quick analyses of quite huge total area.

For the very significant and independent studies on the oscillations of mainly organic groups one can use the Raman microscope, e.g., a Renishaw inVia Reflex spectrometer, equipped with a Leica stereoscopy microscope coupled with a CCD camera for visualization and registration of an image [13].

The atomic force microscopy (AFM) imaging and measurements were carried out using a Nanoscope IIIa device (Digital Instruments-Veeco Company, USA). For the micromechanical measurements, the suitable attachments were necessary, i.e. the nanoindenters enabling the investigation of the microhardness and the nanoscratch facility used for the measurement of friction coefficients [14]. Micromechanical mapping is also possible [15]. Although AFM belongs to the category of nanoinstruments, the micromechanical measurements with nanoindenters are associated with the damage on a surface $\approx 4 \mu \mathrm{m}^{2}$ and we are back in the micrometer fields.

All microanalytical data collected should be related to the topographical details on a sample. It is executed easily when EPMA is used for the collection of the chemical data. In this case the image is obtained using backscattered or secondary electrons. In other cases, the use of a good optical microscope (e.g., Eclipse 400 manufactured by Nikkon Europe) equipped in the image analysis software is essential. 


\section{Some results \\ 3.1. First example}

The analysis of composite petrified wood from Germany is a good example of the verification of the old improper knowledge about the object and establishing new and wider information. The examined wood fragments came from Germany and were of unidentified age. It was embedded in the resin since the original fragments were very fragile. Wood was mineralized in two phases, one of them was deposited in the cell walls, and the second one in the cell lumens. The mineralogical characterization revealed that both substances were iron compounds hematite and siderite. The SEM/EPMA (SEM - scanning electron microscopy) analysis was carried out and the obtained results delivered information inconsistent with that obtained from mineralogical characterization (Fig. 1). In the cell walls the iron was detected. In the lu-
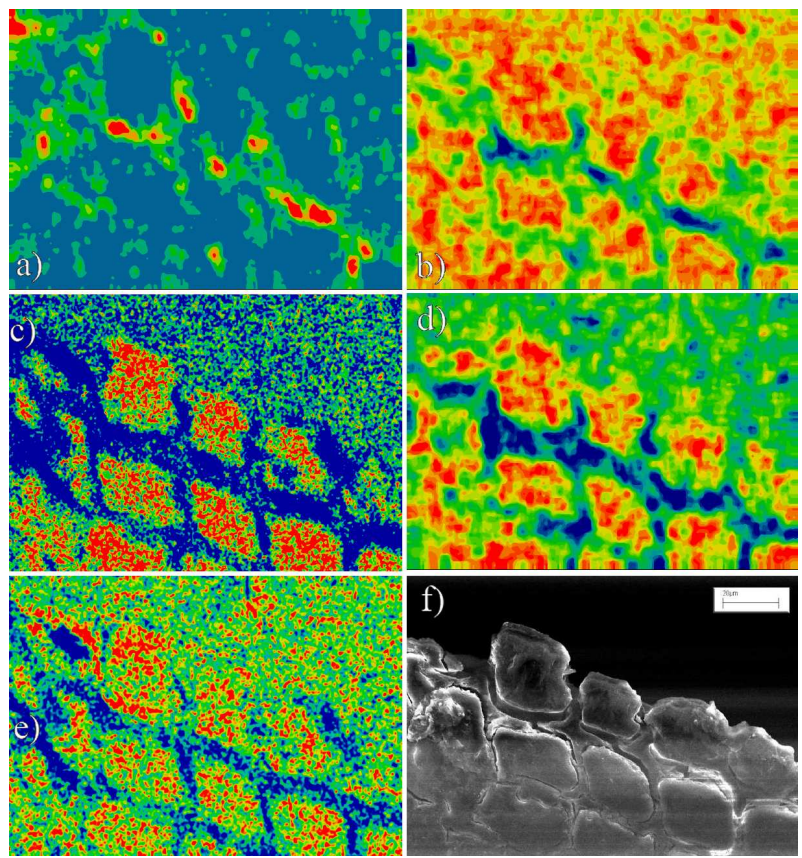

Fig. 1. Elemental mappings for composite petrified wood from EPMA, for: (a) $\mathrm{Fe} K_{\alpha_{1}}$; (b) Ca $K_{\alpha_{1}}$; (c) $\mathrm{O} K_{\alpha_{1}}$; (d) $\mathrm{S} K_{\alpha_{1}}$; (e) $\mathrm{C} K$; (f) backscatter electron image. Scale bar equal to $50 \mu \mathrm{m}$.

mens - Ca, S, O and $\mathrm{C}$ were registered. The existence of an iron-bearing phase in the lumens was excluded; hence the presence of siderite was questioned and the mineralogical composition was still ambiguous. The questions appeared what kind of an iron compound is inside the cell wall and what compounds are located inside lumen - the data suggested the presence of calcite, gypsum or both of them. The crystallographic mapping carried out using MAXIM in HASYLAB explained the situation. Two separate phases of hematite and gypsum were detected and strictly located - hematite in the wall and gypsum in the lumen (Fig. 2). Carbon inside the lumen
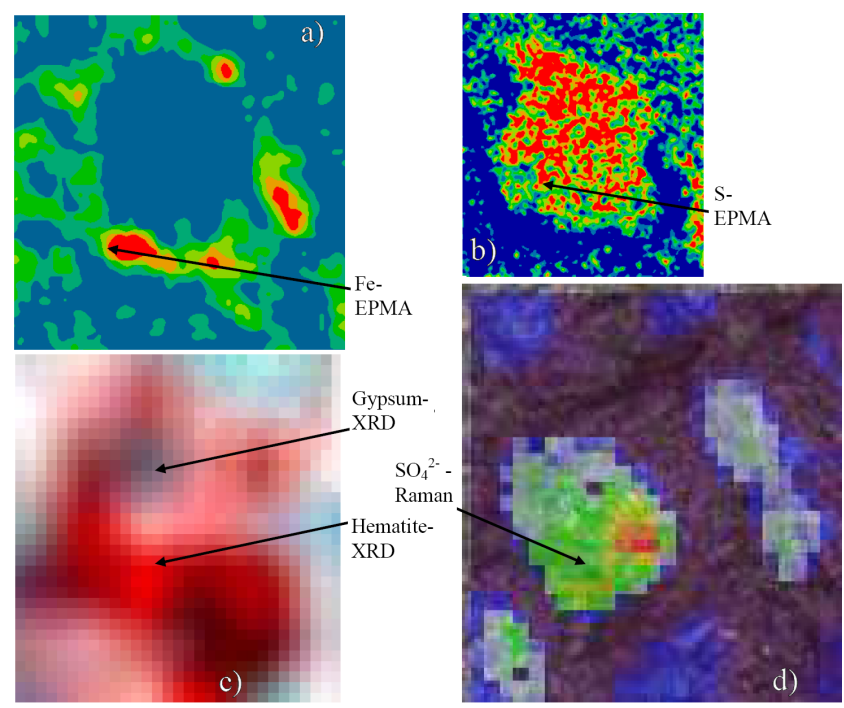

Fig. 2. Comparison of the imaging of the details of petrified wood cell: (a) Fe in the wall, from EPMA; (b) sulfur in the lumen, from EPMA; (c) hematite in the wall and gypsum in the lumen from MAXIM diffraction mapping; (d) $\mathrm{SO}_{4}^{2-}$ oscillation in the lumen, from the Raman microscope.

originated from the resin. Yet it was possible to notice in Fig. 1e that in the middle of the wall zone, on a border between two cells, there is a very thin strip of carbon. We supposed that this carbon is perhaps a component of primordial organic matter, preserved in the petrified wood. We analyzed this fragment very carefully with a Raman spectrometer. Some bands characteristic of lignin or its remains were detected, e.g., the band at $1605 \mathrm{~cm}^{-1}$ attributed to stretching of the benzene ring, characteristic in a lignin. The results are shown in Fig. 3. Thus, the

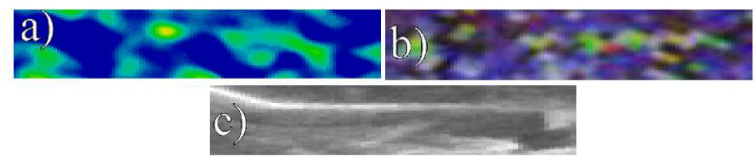

Fig. 3. (a) Thin strip of carbon in the zone of wall of the petrified wood cell, observed by EPMA (C $K$ line); (b) the same strip observed in Raman, lignin - band at $1605 \mathrm{~cm}^{-1}$ — stretching mode of the benzene ring; (c) optical observation of the same strip.

Raman microscopy enabled to supplement the knowledge of the material, proving that it is supported on the organic template belonging to the primordial cell structure of the wood. Surprisingly we can identify the location of this template mirrored in the optical image.

Figure $3 \mathrm{c}$ shows that the strip is clearly observed in optical image of the cells. It is confirmed in Fig. 4, where the Raman presentation of the lignin zone is compared with the deviations in the gray scale levels of an optical image indicating the location of the organic matter. This result finally seems to be trivial, but without carrying out 


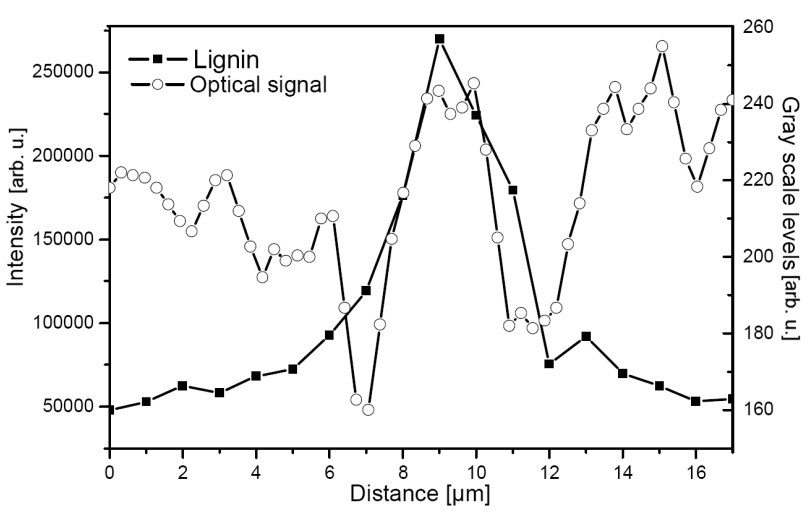

Fig. 4. Comparison of the line scan for the band at $1605 \mathrm{~cm}^{-1}$ - stretching mode of the benzene ring with the scan of the gray scale levels in the transverse section of the two neighboring cell walls in petrified wood.

previous studies using EPMA and Raman we would not be able to explain what this change in the coloration of the preserved cell wall means. Moreover, if we know the composition of the coexisting phases, we can construct the Eh-pH diagram [16] (Fig. 5) showing the field conditions in which these minerals are formed and can coexist, and thus indicating the $\mathrm{pH}$ and Eh conditions that have to be applied to mimic this process of biomineralization. Here, Eh is called activity of electrons and is defined in an analogous way as the well known proton activity term $\mathrm{pH}$, i.e. the negative logarithm of the activity.

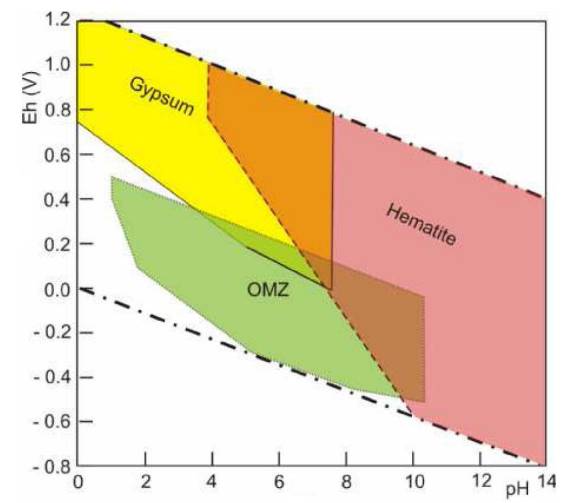

Fig. 5. Eh-pH diagram for three co-existing phases in the cell of petrified wood: gypsum, hematite and lignin remains.

\subsection{Second example}

Human teeth were investigated so intensively as hardly any other biomaterials. However, there is still a field for further studies. Over last years, the small slit (up to $25 \mu \mathrm{m}$ wide, often much narrower) between the enamel and dentin phase attracted the great attention. The popular abbreviation for that zone is dentin-enamel junction (DEJ). It is responsible for assuring the proper contact between the phases mentioned and for preventing the expansion of cracks [17-19]. The structure of DEJ may be determined only with the use of several sophisticated techniques. Here, we present the results of our delimitation of the DEJ by EPMA. The drop in the intensity of P $K_{\alpha_{1}}$ line (optionally $\mathrm{Ca} K_{\alpha_{1}}$ ) indicates the end of the enamel while the increase in the intensity of $\mathrm{C} K$ line shows the beginning of the dentin zone. With the permission of S. Marshall from the Department of Preventive and Restorative Dental Sciences, University of California, San Francisco, USA we tailored their micromechanical data to our chemical data and the result is shown in Fig. 6. The accordance is excellent. In Fig. 7, the DEJ

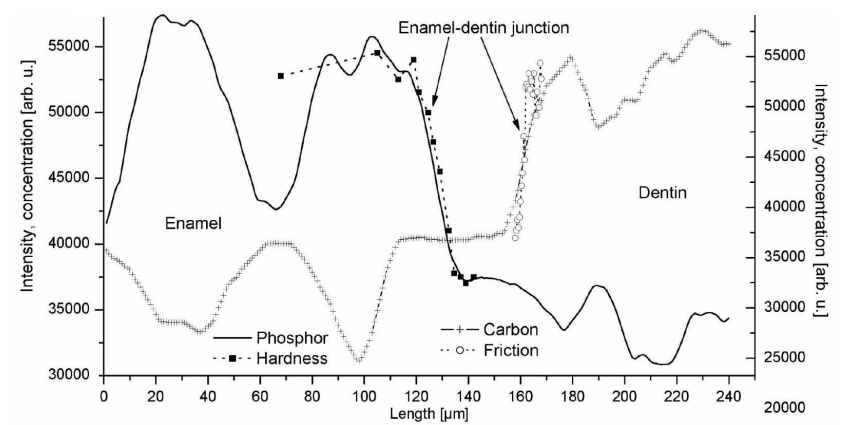

Fig. 6. The boundaries of DEJ zone delimited with the EPMA and micromechanical measurements. P - solid line; C — solid line with crosses; hardness - dotted line with solid squares; friction - dotted line with open circles. The microhardness and microfriction results were accommodated from Marshall et al., with permission.

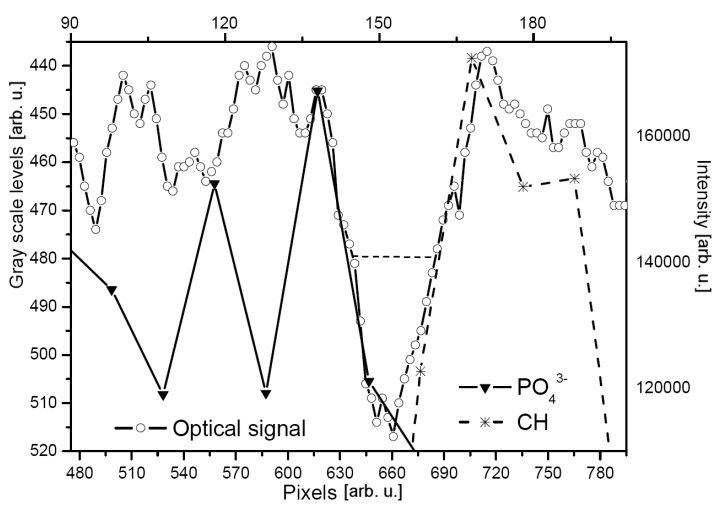

Fig. 7. The boundaries of DEJ zone delimited with the Raman and optical measurements. $\mathrm{PO}_{4}^{3-}$ — solid line with full triangles; $\mathrm{CH}$ - dotted line with crosses; optical profile - solid line with open circles.

is delimitated by the comparison of the optical signal and profiles of two signals from the Raman microscopy: $\mathrm{PO}_{4}^{3-}$ oscillation for the enamel and oscillation of the organic group $\mathrm{CH}$ from collagen for the dentin. Once again, the correlation is excellent. And finally, the comparison of the DEJ images as revealed by optical measurements and by micromechanical indicators is shown in Fig. 8 . 


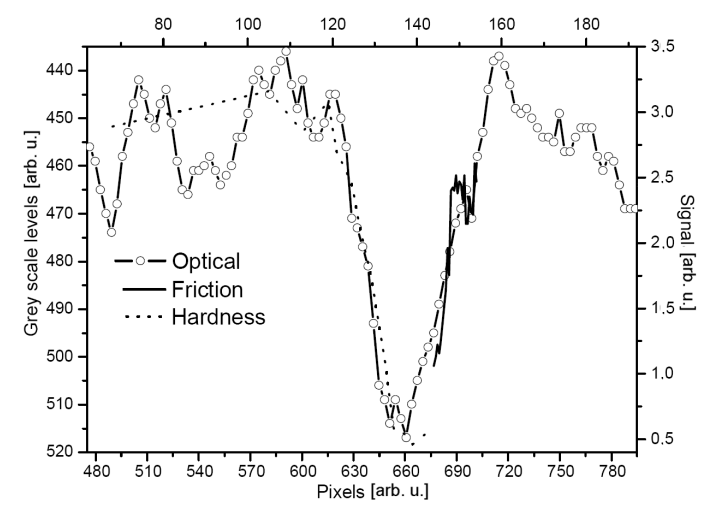

Fig. 8. The boundaries of DEJ zone delimited with the optical and micromechanical measurements. Optical profile — solid line with open circles; hardness dotted line; friction — solid line.

It is striking that the optical representation seems to be most accurate and it reveals all the chemical and micromechanical features of the DEJ in a perfect way. It is a conclusion of great significance. In dental practice the only possible way of observation is optical observation, other instruments are neither tailored nor safe for use in dental surgeries, and it appeared to be most accurate and reliable. But the knowledge about the informative content of an optical image is only "a posteriori" one, after making all other measurements.

\subsection{Third example}

Ammonites, belonging to mollusks, are among the most important and beautiful groups of extinct marine animals. They lived over $300 \mathrm{My}$ from Late Silurian up to $\mathrm{K} / \mathrm{T}$ great extinction. Their beautiful mussels are the perfect index fossils but over last years their construction also attracted the attention [20]. Nevertheless, the construction of their shells is still not fully recognized and the possibility of discovering the presence of ancient organic material is not very clear. We examined an Ammonitida, a fossil from the Callovian period (165-161 My) dug out in Kasimov in Riazan province in Russia. The analyses were carried out using a XRF spectrometer in line $L$ at HASYLAB. Figure 9 presents a mussel wall as revealed by the location of $\mathrm{Ca}$, surrounded by the zone of Fe. In reality, it was even more complex, since from one side a zone enriched in $\mathrm{Zn}$ touched the shell wall. As it was discovered by the combination of XRF and X-ray diffraction (XRD), the zone with $\mathrm{Ca}$ was composed of calcite while the surrounding area with $\mathrm{Fe}$ was built of pyrite. Moreover, the internal variations of the calcite amount in the wall could be detected by the optical signal (Fig. 10a). The similarity of the wall construction as revealed by XRF ( $\mathrm{Ca} K_{\alpha_{1}}$ line) and Raman $\left(\mathrm{CO}_{3}^{2-}\right.$ oscillations) is striking (Fig. 10b). In the middle of calcite zone we detected the remains of archaic organic matter (at least $161 \mathrm{My}$ old) by means of the Raman mapping (Fig. 10c). Only spatial distribution of the $\mathrm{CH}$ group is
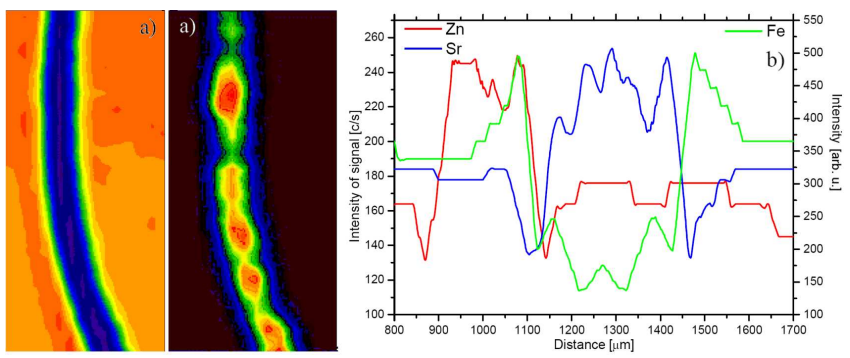

Fig. 9. (a) Mappings of Fe (left) and Ca (right); (b) linear scans imaging of the ammonite wall (Sr), total surroundings (Fe) and peculiar zone enriched with $\mathrm{Zn}$ (result of selective adsorption process?).

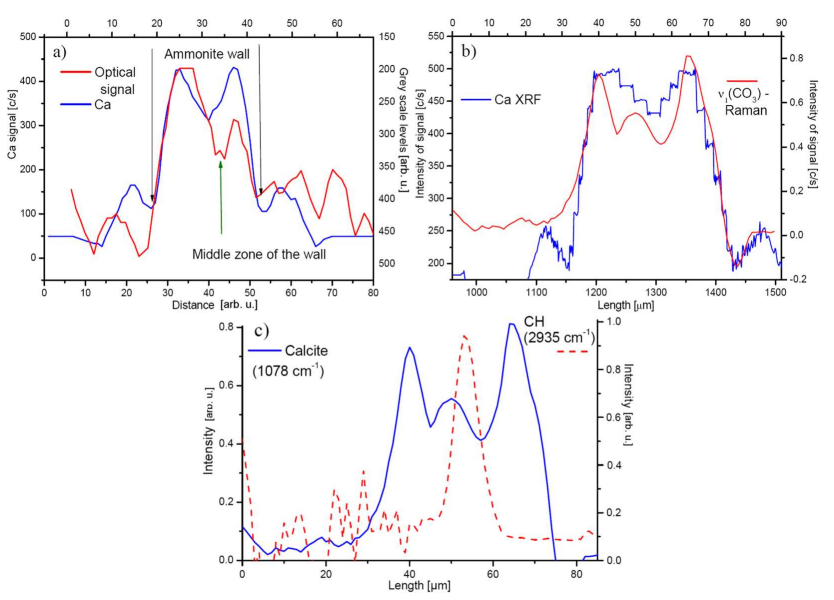

Fig. 10. (a) Comparison of chemical imaging of ammonite wall (Ca from XRF measurement) with the optical profile; (b) near identity of wall imaging by XRF and Raman measurements; (c) detection of archaic organic matter inside the wall by the Raman technique CH line.

presented here but different amide groups were detected as well (not shown here). Once again, although an optical image delivers information, only XRF and Raman measurements are able to explain it.

\section{Discussion}

The question of parallel mapping, scanning (and parallel tomography) is of increasing importance for the complete characterization of patterned objects. The need for such an approach results from the fact that we do not know a perfect instrument. Thus, various supplementary measurements must be undertaken to obtain comprehensive knowledge about biomaterials. We showed that the collection of the consecutive or parallel measurements is essential in two cases:

- to confirm in an independent way the correctness of the results obtained up-to-now;

— to deepen the level of understanding. 
The first case was well illustrated by two examples in our studies. The distribution of inorganic calcite is well observed by the XRF and confirmed by the Raman determination of the carbonate group; independent results match perfectly each other. The same level of confirmation is illustrated in studies on the DEJ slit in teeth. We have possible different combinations of methods (EPMA + micromechanical studies using attachments to AFM; micromechanical + optical) describing the DEJ in a precise way. Some of the combinations give us somewhat trivial qualitative information, e.g. about coupling between the contents of apatite and hardness. This information, however, if treated from the quantitative side is not trivial — we learned that the $5 \%$ drop in the $\mathrm{CaO}$ content corresponds to the $4.5 \%$ in the $\mathrm{P}_{2} \mathrm{O}_{5}$ content and it translates to $2.75 \mathrm{GPa}$ drop in the hardness and $50 \mathrm{GPa}$ drop in the elasticity modulus. The next qualitative information is not trivial: the micromechanical changes can be perfectly coupled with the changes in coloration of a tooth around and inside DEJ.

The desire to deepen the knowledge is obvious. The studies on the composite structure of the cells in the petrified wood allowed first of all to correct the erroneous information from mineralogists about the presence of siderite in the studied sample. The EPMA investigations showed clear distribution of the elements and suggested the possible phases but results were still inconclusive. Only further investigations by XRD (mappings in this case) made the situation clear and the crystallographic phases were precisely identified and located.

The careful observation of the photos and mappings is essential to extract all the abilities of such studies. For example, we noticed in Fig. 1e (EPMA carbon mappings of petrified wood) that there are the separate thin lines enriched in carbon in the zone, where the cells neighbor each other (Fig. 3). It corresponded to the so-called middle lamella in contemporary wood. Our suggestion was that it could be the remains of the primordial organic matter. It could not be confirmed with the electron microprobe alone. Yet in the Raman studies the bands which could be attributed to the lignin or its derivatives were detected easily. Later we noticed that these thin zones could be observed even in an optical image of the cells. But this knowledge was typical "a posteriori" information.

Very similar situation was observed in studies on ammonite walls. We observed inside the wall the very characteristic saddle in the calcium signal (Fig. 10a). It was confirmed in the optical signal and by the Raman measurements (Fig. 10b). The same Raman measurements gave us the answer to the question what is inside the saddle. There were once again the remains of the primordial organic matter, over $160 \mathrm{My}$ old. Of course, now we understand that it was shown by this small detail in the optical image. Once again, this knowledge is "a posteriori" one.

\section{Conclusions}

We used several microprobes to get the comprehensive knowledge of several important patterned biomineral samples. The methods were: EPMA, $\mu$-XRF, $\mu$-Raman and AFM with the micromechanical attachments. All the results were always related to the images from an optical microscope or SEM. It turned out that the optical image is richer than it seemed at first sight but the information was undefined without additional studies. The investigations by the use of different microprobes were complimentary when were performed on the same detail (e.g., the detection of calcite zone in parallel by XRF and Raman for ammonite) or supplementary when we looked for new information (e.g., the detection of the elements and their location in petrified wood by EPMA supplemented by the detection of phases and their location by MAXIM). If the richness of the information collected is considered, the Raman method seems to be unsurpassed but it is plagued by many disadvantages (e.g. disturbances by the luminescence, scattering, difficulties in quantification). Hence even applying this method does not mean that one can avoid the necessity of using supplementary methods.

We can advice the combinations of:

- $\mu$-XRF with $\mu$-XRD, ideal for mineralogical work;

- EPMA with Raman, ideal for the inorganic + organic biomaterials;

- XRF with AFM + nanoindenters, ideal for coupling micromechanical and composition examination;

- SEM with AFM, ideal for parallel visualization of surfaces, with EPMA adding chemical knowledge of surface to the roughness estimation by AFM [21].

Finally, a technical remark. If one wants to take full advantage of such measurements, the construction of a portable and very precise movable sample holder is essential. Only then we can fix a small fragment of a sample in the same location and orientation and compare results from different methods accurately. Similarly, if we have two coupled systems for focusing (as in the Raman microprobe, focusing of laser beam on the sample coupled with focusing of the image from an auxiliary microscope), then both focus points should be the same points.

\section{Acknowledgments}

The authors would like to express their deep gratitude for Professor Sally Marshall from the Department of Preventive and Restorative Dental Sciences, University of California, San Francisco, USA for her permission allowing us to compare our chemical and optical results with her and her team micromechanical data. Acknowledgments to Dr. P. Buurman, Soil Science and Geology, Dept. of Environmental Sciences, Wageningen University, Holland for giving us the very interesting sample of German petrified wood. 


\section{References}

[1] S. Mann, Biomineralization. Principles and Concepts in Bioinorganic Materials Chemistry, 1st ed., Oxford University Press, Oxford 2001, p. 198.

[2] A. Kuczumow, J. Alloys Comp. 362, 71 (2004).

[3] On Biomineralization, Eds. H.A. Lowenstam, S. Weiner, 1st ed., Oxford University Press, Oxford 1989, p. 7.

[4] Biomineralization. From Biology to Biotechnology and Medical Application, Ed. E. Baeuerlein, 1st ed., Wiley VCH, Weinheim 2000, p. 294.

[5] S.A. Wainwright, W.D. Biggs, J.D. Currey, J.M. Gosline, Mechanical Design in Organisms, 2nd ed., Princeton University Press, Princeton 1982, p. 423.

[6] A. Kuczumow, P. Chevallier, Chul-Un Ro, P. Wajnberg, J. Kalita, J. Siurek, Microchim. Acta 137, 173 (2001).

[7] G. Falkenberg, O. Clauss, A. Swiderski, Th. Tschentscher, X-Ray Spectrom. 30, 170 (2001).

[8] S. Lebed, M. Cholewa, Z. Cioch, B. Cleff, P. Golonka, D.N. Jamieson, G.J.F. Legge, S. Łazarski, A. Potempa, C. Sarnecki, Z. Stachura, Nucl. Instrum. Methods Phys. Res. B 158, 44 (1999).

[9] http://hasylab.desy.de/facilities/doris_iii/beamlines/ e6306/index_eng.html.

[10] Th. Wroblewski, A. Buffet, Mater. Sci. Forum 550, 631 (2007).
[11] P. Chevallier, P. Dhez, F. Legrand, A. Erko, Y. Agafonov, L.A. Panchenko, A. Yakshin, J. Trace Microprobe Techn. 14, 517 (1996).

[12] P. Dillman, P. Populus, P. Chevallier, P. Fluzin, G. Beranger, A. Firsov, J. Trace Microprobe Techn. 15, 251 (1997).

[13] J. Nowak, D. Nowak, P. Chevallier, J. Lekki, R. Van Grieken, A. Kuczumow, Appl. Spectrosc. 61, 889 (2007).

[14] S. Habelitz, S.J. Marshall, G.W. Marshall Jr., M. Balooch, J. Struct. Biol. 135, 294 (2001).

[15] J.L. Cuy, A.B. Mann, K.J. Livi, M.F. Teaford, T.P. Weihs, Arch. Oral Biol. 47, 281 (2002).

[16] Atlas of Eh-pH Diagrams - Intercomparison of Thermodynamic Databases, Geological Survey of Japan Open File Report No. 419.

[17] H. Fong, M. Sarikaya, S.N. White, M.L. Snead, Mater. Sci. Eng. C 7, 119 (2000).

[18] K.A. Schultze, M. Balooch, G. Balooch, G.W. Marshall, S.J. Marshall, J. Biomed. Mater. Res. A 69, 286 (2004).

[19] V. Imbeni, J.J. Kruzic, G.W. Marshall, S.J. Marshall, R.O. Ritchie, Nature Mater. 4, 229 (2005).

[20] Y. Dauphin, Int. J. Earth Sci. 91, 1071 (2002).

[21] R. Mroczka, P. Bartosik, Z. Sawłowicz, K. Skrzypiec, G. Falkenberg, J. Wójcik, G. Żukociński, A. Kuczumow, Thin Solid Films 516, 8029 (2008). 